\title{
An improved technique for the simultaneous measurement of nasal and oral respiration
}

\author{
Christopher L. Keall, B.D.S., M.S.,* and \\ Peter S. Vig, B.D.S., Ph.D., D. Orth., F.D.S.R.C.S.(Eng.) ${ }^{\star \star}$ \\ Ann Arbor, Mich.
}

\begin{abstract}
A technique is described to record and measure both the nasal and oral components of respiratory aiflow. The method is a modification of a previously reported technique, ${ }^{1}$ and represents an improvement in terms of accuracy, speed, convenience, and facility in both the acquisition and analysis of a large set of data per subject. The equipment and associated computer configuration permits a temporal characterization of inspiratory and expiratory parameters of both nasal and oral airflow, nasal airway resistance computation at predetermined flow rates, and the calculation of estimates of the minimum cross-sectional area of the nasal air passage. (AM J ORTHOD DENTOFAC ORTHOP 1987;91:207-12.)
\end{abstract}

Key words: Respiration, nasal airway resistance, nasal/oral ratio, nasorespiratory function

$T_{\text {he relationships among nasorespiratory }}$ function, the cause of malocclusion, and the efficacy of diagnostic procedures or various forms of therapy for impaired breathing are still unclear. Despite the lack of objective data, many clinicians in both orthodontics and the medical specialties of allergy and otorhinolaryngology recommend a variety of treatment modalities for suspected nasal obstruction. This is particularly true in the case of children who have dentofacial morphologic features that historically have been ascribed to environmental influences on growth and development. ${ }^{2-6}$

The so-called long-face syndrome or "vertical maxillary excess," which a generation ago was called the "adenoidal facies," does present a difficult challenge to the orthodontist, irrespective of its true cause. Patients with a high Frankfort mandibular plane angle and a long lower facial height frequently also have lip separation at rest (or lip "incompetence"). This, however, does not necessarily mean that mouth breathing exists, or that respiration is either difficult or impossible via the nasal airway. Lip incompetence is not synonymous with "mouth breathing," but rather it is to be considered in the majority of children as a normal stage of growth and a reflection of the asynchrony between the vertical skeletal development of the lower facial height and the growth of the soft-tissue integument. ${ }^{7}$

Given the alternatives of either accepting the hy-

From the University of Michigan.

Supported by NIDR Grant DE 06881

*Research Fellow.

** Chairman and Professor, Orthodontic Department; Research Scientist, Center for Human Growth and Development. pothesis of an environmentally induced adaptation for mouth breathing or facing the reality of our present state of ignorance, it is not surprising that some clinicians prefer a doubtful certainty to a certain doubthence, perhaps the willingness to embrace the views of those who recommend some positive, but possibly unjustifiable, interventions such as adenoidecromy, antiallergy treatment, or rapid maxillary expansion to "improve" respiration for orthodontic rcasons. Unfortunately, we have no evidence from any properly designed, prospective clinical trials to suggest that orthodontic treatment is made easier, or better, either in terms of short- or long-term prognosis by any procedures aimed at improving nasal respiration. No randomized, prospective studies with specified end points and suitable controls have been reported to date.

Our respiratory studies are aimed at providing objective quantitative information on the physiologic parameters that characterize oral and nasal respiratory behavior. Although a considerable literature now exists on nasal resistance and variations in nasal airflow rate, this in itself does not provide an adequate basis to determine the extent to which any given person is a mouth breather, nose breather, or somewhere in-between. For this reason, work has been in progress for some years to develop a technique to establish the ratio of nasal/oral airflow, or the "respiratory mode," as a quantifiable index of nasopharyngeal obstruction or patency.

A Simultaneous Nasal and Oral Respirometric Technique (SNORT) was reported in 1981:8 The presently used method is a considerably refined and im- 


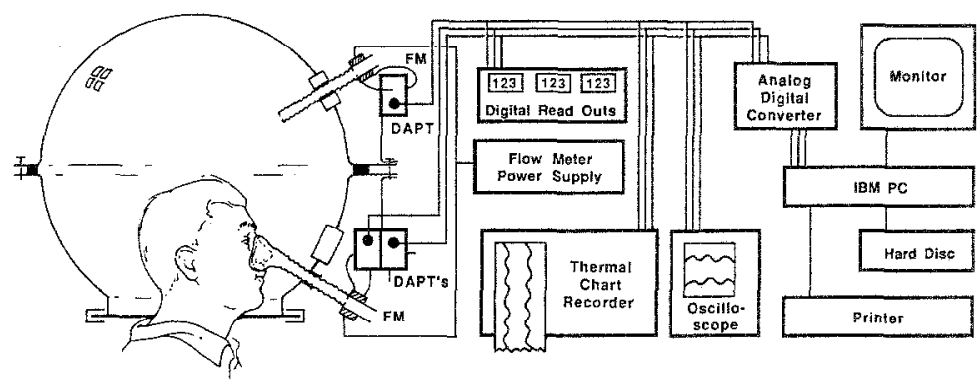

Fig. 1. Schematic diagram of SNORT system. FM, Flowmeter. DAPT, Differential air pressure transducer.

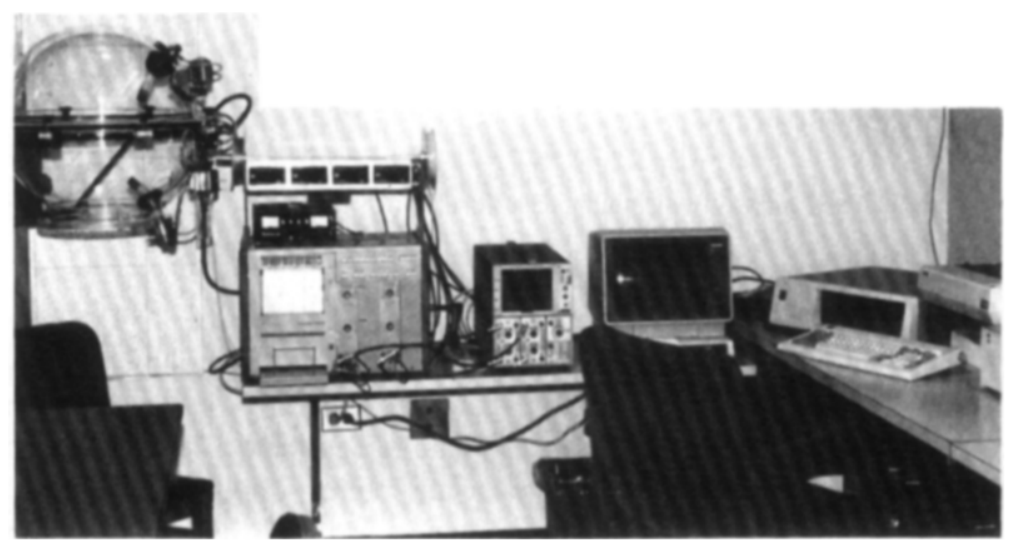

Fig. 2. SNORT assembly. Shown from left: chamber, digital readouts, flowmeter power suppiy, chart recorder, oscilloscope, IBM PC, and peripherals.

proved modification of SNORT. Our equipment and procedures have both been modified specifically to reduce the following problems of the original SNORT:

1. High potential for leakage

2. Time-consuming elaborate assembly/disassembly

3. Large airway dead space in long tubing runs

4. Airway resistance in plastic tubing runs and flowmeters

5. Possible artifacts caused by use of two-way valves at the oral and nasal ports

6. High compliance of neck seal (moved with respiration)

7. Subject required to wear restrictive headgear to support nasal mask

8. Somewhat claustrophobic experience for subject

\section{DESCRIPTION OF THE NEW APPARATUS}

The modified SNORT apparatus is shown in Figs. 1 through 3 . The subject is seated comfortably with the head inside the Plexiglas SNORT chamber. The chamber is made up of an upper and a lower half, each with a peripheral flange. These seal together with a rubber gasket using eight quick release screw fasteners forming a completely airtight joint. The lower half has a circular opening at the bottom with a flange around the periphery.

The subject wears a four-layer collar to form a seal between the neck and the lower chamber. The collar consists of (1) two neoprene disks with off-center holes that stretch over the subject's head and fit snugly around the neck, and (2) a semirigid plastic disk with a slightly larger off-center hole and a radial cut in it-which allows it to be fitted around the neck without touching it-between the two neoprene disks to reduce their compliance. There is a Plexiglas ring that sandwiches the other three layers against the flange around the bottom opening in the chamber. Using four Teflon spring clamps, this assembly is quickly and easily secured to the base of the chamber.

The chamber is supported on a counterbalanced bracket that allows the entire apparatus to be raised or lowered over the subject's head and adjusted for height with the subject comfortably seated.

Once inside the SNORT chamber, the subject pushes his/her face gently against a nose mask connected to a semiflexible concertina anesthetic tubing 


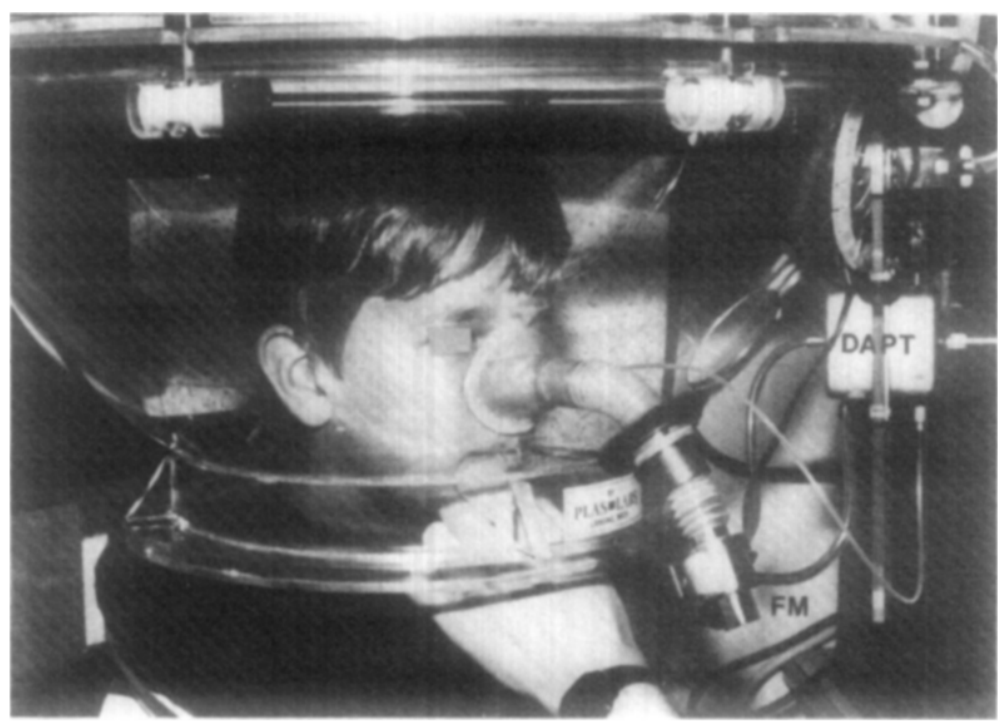

Fig. 3. Patient in chamber showing pross fit into nasal mask attached by flexible tubing to flowmeter (FM) and differential air pressure transducer (DAPT).

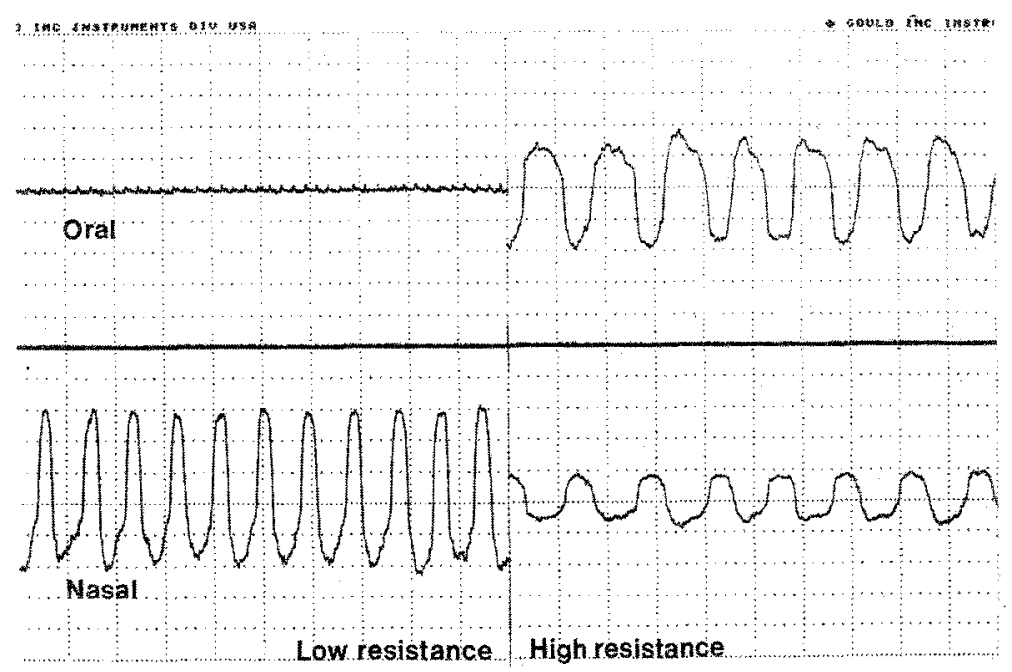

Fig. 4. Composite of oral and nasal flow rate on the strip chart record. Upper trace is oral flow rate, middle trace is oronasal differential pressure (inactive during oronasal recordings), lower trace is nasal flow rate. Left side shows a patient with a low nasal resistance-negligible oral component (notice the heartbeat recorded as faint noise) and almost total nasal breathing. Right side shows a patient with a high nasal resistance-predominantly oral breathing but still with a considerable nasal component.

that passes through a port in the lower chamber wall. This is coupled to a heated bidirectional flowmeter (FM) connected to a differential air pressure transducer (DAPT) that is attached to the lower half of the chamber. The port in the chamber wall allows the anesthetic tubing to be moved in and out to adjust the position of the nose mask for each subject. The same arrangement without a nose mask is attached to the upper half of the chamber to measure oral flow.

A range or variety of nose masks of different shapes and sizes has been made on models obtained from a series of earlier subjects; the closest fit is selected for each subject. The nose masks are made of mouth guard material formed on a model with a modeling clay spacer applied to give approximately $5 \mathrm{~mm}$ clearance around the nose with a $5 \mathrm{~mm}$ wide flange touching the face around the periphery. A self-adhesive window sealing strip made of soft rubber is applied to the flange to provide a flexible seal around the nose of the subject. This makes the nose mask airtight around its seal. Fre- 


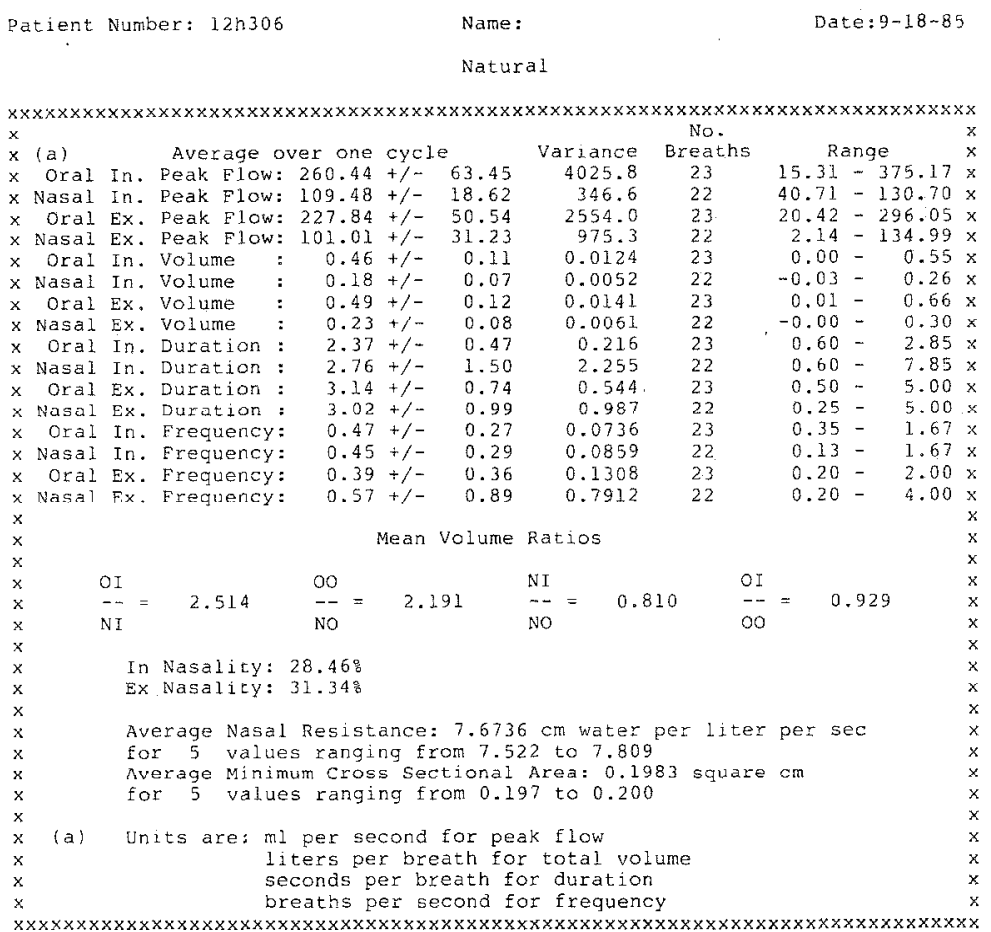

Fig. 5. Specimen computer printout from a recording session. In., Inspiration. Ex., Expiration. Ol, Oral in. OO, Oral out. NI, Nasal in. NO, Nasal out.

quent leakage tests are made to verify that all junctions are properly sealed and that leakage is controlled throughout the entire apparatus.

The lower half of the chamber has a second port in front of the subject's face and also supports an extra DAPT to enable nasal resistance measurements to be made without removing the subject from the chamber. The second port can also be used to introduce measured quantities of air to calibrate the apparatus and is used during leakage tests.

A variable power supply is used to supply current to the heating elements in both of the flowmeters to prevent condensation. The electrical connections (heating power supply, DAPT power supply, and signal output) for the upper half of the chamber are all made through one connector to the lower half of the chamber to enable easy removal of the upper chamber.

The electrical signals from the three DAPTs, which monitor oral airflow, nasal airflow, and nasal resistance in terms of pressure differentials, drive the following equipment: (1) three digital readouts, (2) an oscilloscope, (3) a thermal chart recorder, and (4) an analogdigital converter that in turn feeds into an IBM PC computer.

The use of bidirectional flowmeters and DAPTs permits both oral and nasal inspiration and expiration to be measured simultaneously with only two channels. This decreases the number of calibrations required and simplifies data display and recording. In addition, there is no longer a requirement for the large two-way valves that the original SNORT used, thus reducing the dead space. Similarly, taking the nasal and oral tubes out through the front of the chamber reduces the dead space because the tube lengths can thus be signiticantly reduced. As the nasal and oral tubes take a straight path, it has been possible to use a single, larger diameter tube, thereby reducing the inherent resistance to airflow within the system. Flexibility is retained by making use of a lighter concertina anesthetic tubing whose anteroposterior position may be adjusted by means of its tight friction fit through the brass port in the chamber wall.

The addition of the DAPT for nasal resistance determination allows a subject to remain in the SNORT chamber through a series of tests that record both flow and resistance. This saves considerable time. During nasal resistance measurements, the top half of the chamber is removed to provide access to the subject and to create a sense of freedom.

During a typical flow measurement session, which lasts about 3 minutes with only 125 seconds of data collection, temperaturc inside the chamber increases by approximately $0.3^{\circ} \mathrm{C}$. According to the Charles law, within moderate ranges of temperature, the volume assumed by a given mass of gas is directly proportional to the corresponding absolute temperature measured on the Kelvin scale. Because our experimental work 
is carried out in the region of $300^{\circ} \mathrm{K}$, a change of $0.3^{\circ} \mathrm{C}$ would give only a $0.1 \%$ error per breath and a cumulative oral expiratory surplus of $50 \mathrm{ml}$ over perhaps 30 respiratory cycles. It is obvious that very large temperature changes inside the apparatus would be required to substantially affect our results in terms of airflow.

The digital readouts and the oscilloscope provide a convenient visual means to determine when a subject has reached a steady state of respiration or has mastered the breathing technique required for nasal resistance measurement. This is used to monitor subjects to avoid the collection of spurious or artifactual data. The chart recorder provides a permanent graphic representation of each breathing recording session. This has proved to be a valuable visual aid to compare patients or to demonstrate differences among various subjects' breathing patterns as shown in Fig. 4 .

The IBM Personal Computer is configured with the following items: $640 \mathrm{~K}$ of internal memory, 2 floppy disk drives, and a 15 megabyte external hard disk drive. In addition, the computer is fitted with a Tecmar Labmaster board* that connects to the external analog to digital converter $(A D C)$. The software used to control the Labmaster board and ADC is the LabPac package, which is a series of machine language routines designed for collecting data and controlling the ADC. Custommade programs in either BASIC, FORTRAN, or PASCAL are required to manipulate the LabPac routines and to handle the raw data.

For oral/nasal ratio studies, the computer is programmed to record nasal and oral differential pressures 20 times per second for 125 seconds. These data are then coverted into flow rates and stored on a floppy disk. On completion of a series of recordings, the computer will analyze the data and determine the following for each of the 125-second recordings: average, standard deviation, variance, sample size (that is, the number of data points recorded), and range of values obtained for each of peak flow, volume, duration, and frequency for both oral and nasal inspirations and expirations ( 80 results in all); mean volume ratios for oral/ nasal inspiration and expiration, and nasal and oral inspiration/expiration; and nasal component expressed as a percentage of the inspiration and expiration volumes. For an example of the printout obtained for a typical recording session, see Fig. 5.

Nasal resistance is measured by means of a previously reported technique ${ }^{9}$ and calculated by the formula

$$
\mathbb{R}=\mathbf{P} / \mathrm{V}
$$

*Techmar Incorporated, Personal Computer Products Division, Cleveland, Ohio. where $R$ is the resistance in centimeters of water per liter per second, $P$ is the pressure differential between the posterior oropharynx and the outside of the nose in centimeters of water, and $\mathrm{V}$ is the nasal fiow rate in liters per second. Minimum cross-sectional area is calculated from the same values of $P$ and $V$ by a previously derived formula ${ }^{10}$ :

$$
\text { Area }=\mathrm{V} / \mathrm{k}[2(\mathrm{P}) / \text { density of air }]^{1 / 2}
$$

where constant $k=0.65$, the density of air $=0.001$ $\mathrm{g} / \mathrm{cm}^{3}$, and the minimum cross-sectional area is expressed in $\mathrm{cm}^{2}$.

For nasal resistance studies, the computer is programmed to calculate and display the nasal resistance after each determination and to give the operator the choice of whether to save that value or discard it. The computer analysis of these data drops the highest and lowest values and determines the average and range for both nasal resistance and minimum cross-sectional area. Our standard practice is to discard negative values of nasal resistance that are produced as an artifact when subjects create negative pressures by obstructing the oral catheter or the pharynx. We discard very high nasal resistance values (above 7) unless, after checking for obstruction of the nose by the mask, they are reproduced. We save 7 nasal resistance values so that the statistical analysis is the result of five representative nasal resistance determinations. It is possible to set the flow rate at which nasal resistance is determined at any value between 50 and $500 \mathrm{ml} / \mathrm{sec}$.

\section{ERRORS OF THE SYSTEM}

Possible leakage of the chamber is checked by applying a constant flow of air directly to one of the flowmeters and then indirectly through the chamber; in this manner leaks can be isolated and reduced to less than $1 \%$. The differential air pressure transducers have a potential error of $2 \%$, which is effectively eliminated for flow rate measurements by zeroing and calibrating each channel at the start of each recording session. However, this $2 \%$ error will apply to nasal resistance determinations, but this is well below the level of individual patient variability. Calibration itself has an error of $1 \%$. On the electronic side, the analog-digital converter has a $0.05 \%$ error and the computer has an error of up to $0.00005 \%$ per calculation, perhaps up to $1 \%$ in total.

The potential errors within the system are cumulative; thus, the overall error levels are $1.7 \%$ for oronasal flow and $2.6 \%$ for nasal resistance.

\section{DISCUSSION}

Our studies are part of a collaborative project with the workers at the University of North Carolina, under 
the leadership of D. W. Warren. Some of the questions that we address in our laboratory are also being investigated by the North Carolina group, who use an alternative technique for the estimation of respiratory mode. Warren, Hairfield, and Hinton ${ }^{11}$ employ inductive plethysmography in their determination of the oral component of breathing.

Continued collaboration will serve not only to corroborate our findings, but will also help to evaluate the respective utility of the two alternative systems currently available for quantifying oral-nasal respiratory parameters.

The issue of nasorespiratory function in relation to orthodontics is still controversial. ${ }^{12}$ Current clinical concepts are all too often based on subjective opinion, inadequate data, and an incomplete understanding of the physiologic characteristics of the respiratory mode of patients who happen to have malocclusion.

The technique described provides an easy means to quantify associations between nasal resistance to airflow and the degree to which a person is breathing through the mouth or nose. Our preliminary studies suggest that, within a range of values, nasal resistance is not a good predictor of the percentage nasality of respiratory mode. This is contrary to the prevailing views that are expressed in the literature.

Furthermore, changes in resistance to airflow and respiratory mode are being recorded under various physiologic conditions in patients before and after certain orthodontic, orthognathic, and otolaryngologic treatments. These studies, together with evaluations of untreated persons at different ages and with differing physical characteristics, will provide information necessary for the establishment of nonnative standards and an evaluation of the efficacy of therapeutic intervention.

We wish to acknowledge the contributions of Kevin Ankoviak, who developed the computer configuration and soft- ware; Heather Keall, who conducted the clinical trials and developed the protocol; and Dick Miller, who continues to provide technical assistance.

\section{REFERENCES}

1. Gurley WH, Vig PS. A technique for the simultaneous measurement of nasal and oral respiration. AN J ORTHOD 1982;82: 33-41.

2. Linder-Aronson S. Effects of adenoidectomy on the dentition and facial skeleton over a period of five years. Trans Eur Orthod Soc, 1972, 177-86.

3. Holmberg $\mathrm{H}$, Linder-Aronson S. Cephalometric radiographs as a means of evaluating the capacity of the nasal and nasopharyngeal airway. AM J ORTHOD 1979;76:479-90.

4. Quinn GW. Are dentofacial deformities a preventable disease? NC Dent J 1978;61:5-6.

5. Rubin RM. Mode of respiration and facial growth. AMJ ORTHOD 1980;78:504-10.

6. McNamara JA Jr. A method of cephalometric evaluation. AM J ORTHOD 1984;86:449-69.

7. Vig PS, Cohen AM. Vertical growth of the lips: A serial cephalometric study. AM J ORTHOD 1979;75:405-15.

8. Gurley WH III. A technique for the simultaneous measurement of nasal and oral respiration [Thesis]. Chapel Hul, North Carolina: University of North Carolina at Chapel Hill, 1981. pp xi, 180.

9. Watson RM, Warren DW, Fischer ND. Nasal resistance, skeletal classification, and mouth breathing in orthodontic patients. AM J ORTHOD 1968:54:367-79.

10. Warren DW. A quantitative technique for assessing nasal airway impairment. AM J ORTHOD 1984;86:306-14.

11. Warren DW, Hairfield WM, Hinton VA. Inductive plethysmography for objective assessment of mouth breathing [Absiract]. $\mathrm{J}$ Dent Res 1985;64:348.

12. Vig PS. Respiration, nasal airway, and orthodontics: A review of current clinical concepts and research. In: Johnston LE, ed. New vistas in orthodontics. Philadelphia: Lea \& Febiger, 1985;76-102.

Reprint requests to:

Dr. Peter S. Vig

The University of Michigan

School of Dentistry

Ann Arbor, MI 48109-1078 